Article

\title{
Global Stability of a Markovian Jumping Chaotic Financial System with Partially Unknown Transition Rates under Impulsive Control Involved in the Positive Interest Rate
}

\author{
Ruofeng Rao ${ }^{1,2}$ \\ 1 Department of Mathematics, Chengdu Normal University, Chengdu 61130, China; ruofengrao@163.com or \\ ruofengrao@cdnu.edu.cn \\ 2 Institute of Financial Mathematics, Chengdu Normal University, Chengdu 61130, China
}

Received: 22 May 2019; Accepted: 25 June 2019; Published: 28 June 2019

check for updates

\begin{abstract}
The intrinsic instability of the financial system itself results in chaos and unpredictable economic behavior. To gain the globally asymptotic stability of the equilibrium point with a positive interest rate of the chaotic financial system, pulse control is sometimes very necessary and is employed in this paper to derive the globally exponential stability of financial system. It should be pointed out that the delayed feedback model brings an essential difficulty so that the regional control method has to be adopted. In this paper, the author firstly employs impulsive control, regional control, the Lyapunov function technique, and variational methods to derive the stochastically globally asymptotic stability criterion of the economic balance point with a positive interest rate for a delayed feedback financial system with Markovian jumping and partially unknown transition rates. Besides, the mathematical induction method and the proof by contradiction are applied synthetically to deduce the globally exponential stability of the equilibrium point with a positive interest rate for the impulsive financial system without time-delays. Moreover, numerical examples illustrate that under suitable data conditions on the two main criteria mentioned above, the interest rates are positive decimals when the financial system reaches stability, which means better economic significance.
\end{abstract}

Keywords: positive interest rate; chaotic financial system; reduction to absurdity; mathematical induction method; impulse control

\section{Introduction and Preparation}

In recent decades, the following financial mathematical model has received wide attention (see [1-7] and the references therein), which is composed of the production sub-block, currency sub-block, securities sub-block, and labor sub-block:

$$
\left\{\begin{array}{l}
\dot{x}=z+(y-a) x \\
\dot{y}=1-b y-x^{2} \\
\dot{z}=-x-c z,
\end{array}\right.
$$

where $x$ represents the interest rate, $y$ represents the investment demand, $z$ represents the price index, $a$ represents savings, $b$ represents the unit investment cost, and $c$ represents the elasticity of commodity demand. Although the financial system (1) is a definite system, a dynamic test shows that chaos is the intrinsic stochasticity in this definite system, and the intrinsic stochasticity is the irregular dynamical behavior of the system, which is difficult to predict [3-7]. Chaos is a process similar to stochastic phenomena in deterministic systems and a seemingly irregular movement. The financial system is a 
complex non-linear system composed of many factors. In this non-linear system, with the change of system parameters, its motion state may appear as chaotic phenomena, leading to the emergence of financial crisis. Therefore, in this paper, the control of the chaotic financial system is considered.

On the other hand, there is much literature related to dynamical analysis. For example, In [3,4], the dynamic properties of the system (1), such as equilibrium point and bifurcation, were analyzed. One can see from $[3,4]$ that if $c-b-a b c$ is no greater than zero, there is the unique equilibrium point $Q_{0}\left(0, \frac{1}{b}, 0\right)$ in the financial system (1); if $c-b-a b c$ is not less than zero, there exist three equilibrium points $Q_{0}\left(0, \frac{1}{b}, 0\right), Q_{1}\left(\sqrt{\theta}, \frac{1+a c}{c}, \frac{-\sqrt{\theta}}{c}\right), Q_{2}\left(-\sqrt{\theta}, \frac{1+a c}{c}, \frac{\sqrt{\theta}}{c}\right)$ in the financial system (1), where $\theta=\frac{c-b-a b c}{c}$. The following proposition is true:

Proposition 1 ([3,4]). If $c-b-a b c>0, Q_{0}$ is the stable equilibrium point. However, both $Q_{1}$ and $Q_{2}$ are unstable if $b c^{4}+b^{2} c^{3}-2 a b^{2} c^{2}+\left(2 a b-2-3 b^{2}\right) c+3 b=0$.

Besides, the delay feedback dynamics and feedback control of the system [1] were discussed in [5]. The $H_{\infty}$ control problem with external disturbances and input delays of the system (1) was considered in [6]. Chaotic synchronization of the system (1) was studied in [7].

Remark 1. From [8] (Table 1), one can see that chaos appears in the financial system (1) if $c-b-a b c>0$ and $c+a-\frac{1}{b}<0$. For example, if a equals $0.9, b$ is 0.2 , and c equals 1.2 , chaotic behaviors are characterized in the financial system (1) (see Figure 1).

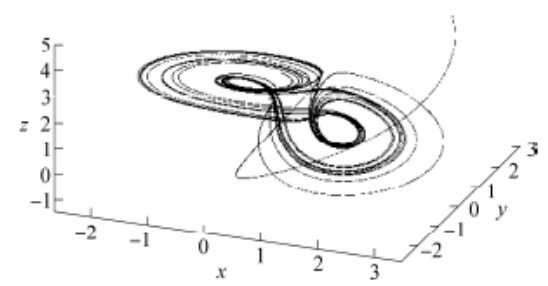

Figure 1. Phase diagram of the financial system (1) for $t \in[0,10,000]$.

Let:

$$
\left\{\begin{array}{l}
X_{1}=x-\sqrt{\theta} \\
X_{2}=y-\frac{1+a c}{c} \\
X_{3}=z+\frac{\sqrt{\theta}}{c}
\end{array}\right.
$$

the financial system (1) is translated into the following system:

$$
\left\{\begin{array}{l}
\dot{X}_{1}=\frac{1}{c} X_{1}+\theta X_{2}+X_{3}+X_{1} X_{2} \\
\dot{X}_{2}=-2 \theta X_{1}-b X_{2}-X_{1}^{2} \\
\dot{X}_{3}=-X_{1}-c X_{3}
\end{array}\right.
$$

where the equilibrium point $Q_{1}\left(\sqrt{\theta}, \frac{1+a c}{c}, \frac{-\sqrt{\theta}}{c}\right)$ becomes the null solution of the system (3). Furthermore, one can rewrite the system (3) in matrix-vector form:

$$
\left\{\begin{array}{l}
\dot{X}=-A X+f(X), \quad t \geqslant t_{0}=0 \\
X\left(t_{0}\right)=X_{0}
\end{array}\right.
$$


where $X^{T}=\left(X_{1}, X_{2}, X_{3}\right), X_{0}=\left(X_{01}, X_{02}, X_{03}\right)^{T} \in R^{3}$, and:

$$
A=\left(\begin{array}{ccc}
-\frac{1}{c} & -\theta & -1 \\
2 \theta & b & 0 \\
1 & 0 & c
\end{array}\right), \quad f(X)=\left(\begin{array}{c}
X_{1} X_{2} \\
-X_{1}^{2} \\
0
\end{array}\right)
$$

In 2017, impulse control on the financial system (1) was discussed in [9]. However, the numerical example of [9] only verified the asymptotical stability of the equilibrium point $Q_{0}\left(0, \frac{1}{b}, 0\right)$ with zero interest rate when the system reaches stable. In fact, the globally asymptotical stability of $Q_{1}$ with the positive interest rate $\sqrt{\theta}>0$ needs to involve the more complex impulse control technique. In this paper, the author will employ the proof by contradiction, the mathematical induction method, and the Lyapunov function method to guarantee the globally asymptotical stability of the equilibrium point $Q_{1}$ of the financial system (1) under the suitable impulse control, i.e., guaranteeing the globally asymptotical stability of the null solution for the following system:

$$
\left\{\begin{array}{l}
\dot{X}=-A X+f(X), \quad t \geqslant t_{0}=0, t \neq t_{k}, k \in \mathbb{Z}^{+} \\
X\left(t_{k}^{+}\right)=X\left(t_{k}\right)=B_{k} X\left(t_{k}^{-}\right), \quad k \in \mathbb{Z}^{+} \triangleq\{1,2, \cdots\} \\
X\left(t_{0}\right)=X_{0}
\end{array}\right.
$$

where $f$ and $A$ are defined in (5) and each $B_{k}$ represents the impulsive parameter matrix. The impulsive time $t_{k}\left(k \in \mathbb{Z}^{+}\right)$satisfies $0<t_{1}<t_{2}<\cdots<t_{k}<\cdots$ with $\lim _{k \rightarrow \infty} t_{k}=\infty$.

Remark 2. The equilibrium point $Q_{1}$ with the positive interest rate $\sqrt{\theta}>0$ is not stable in the financial system (1) under any data of $a, b, c$. Therefore, in this paper, the author has to consider employing the suitable impulse control to secure global stability of $Q_{1}$.

Remark 3. According to the principles of macroeconomics, the interest rate is usually a positive decimal whenever the product market and the money market are in common equilibrium. Therefore, in this paper, the author considers gaining the globally asymptotical stability of the equilibrium point $Q_{1}$ with the positive interest rate, which is more applicable for the real needs of complex financial market realities.

A fact should be pointed out that there is a time lag in dynamic economic variables, for there is a time lag between making economic decisions and the effectiveness of decisions. Usually, economic agents are considered rational $[5,10,11]$. For example, in [5], the following time delayed feedback financial system was introduced:

$$
\left\{\begin{array}{l}
\dot{x}=z+(y-a) x+k_{1}\left(x-x\left(t-\tau_{1}\right)\right) \\
\dot{y}=1-b y-x^{2}+k_{2}\left(y-y\left(t-\tau_{2}\right)\right) \\
\dot{z}=-x-c z+k_{3}\left(z-z\left(t-\tau_{3}\right)\right)
\end{array}\right.
$$

The chaos behavior of dynamic economic system usually implies that there exists intrinsic instability in macroeconomic movement itself, which is further aggravated by the delayed feedback of the financial system in reality (see, e.g., Figure 2).

Remark 4. Time delays bring essential difficulties in deriving the globally asymptotical stability of the equilibrium point $Q_{1}$ with the interest rate. Actually, the methods employed for the system (6) without time delays may be no longer applicable to the delayed system (7).

Remark 5. Although the authors of [12] utilized the impulsive delayed differential inequality to gain the global exponential stability criterion of the equilibrium point $Q_{0}(0,1 / b, 0)$ for a delayed financial system, the methods are no longer applicable to that of $Q_{1}$ with the positive interest rate. 
Remark 6. In order to overcome the double difficulties caused by time delay and positive interest rate, the author has to adopt double control methods: impulse control and regional control.
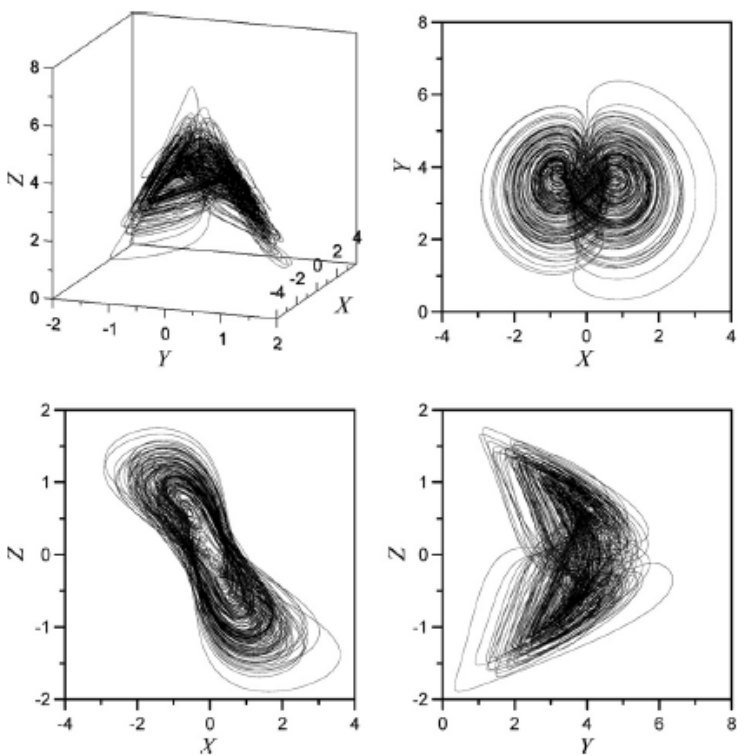

Figure 2. Trajectories for the system (7) where $k_{i}=0.1(i=1,2,3)$ and $\tau=9$.

Recall that impulsive effects of complex dynamic systems have been investigated [13-20]. The impulses may lead to the instability or stability of the system depending on the magnitude of the impulses. impulsive effects may perturb or destabilize the phase trajectories of the systems provided that the magnitude of impulsive strength is greater than one. If the magnitude of impulsive strength is less than one, impulsive effects may stabilize the unstable neural networks. In this paper, the author will consider this class of impulsive control for the delayed feedback model of the financial system (1). On the other hand, time delays bring great difficulties to stability analysis of the equilibrium point $Q_{1}$ with the positive interest rate for the delayed feedback financial system (7). Based on this consideration, the author introduces the regional control method. Recall that the diffusion phenomenon appears really in the neural networks whenever electrons go through asymmetric electromagnetic fields [21-25]. If the financial indicators are related to the region by macro-control, adjusting the diffusion coefficient contributes to the global asymptotic stability of the equilibrium point $Q_{1}$ with the positive interest rate for the delayed feedback financial system. Therefore, in this paper, the author considers the reaction-diffusion model when investigating the delayed feedback financial system.

Besides, recall that the Markov model simulates well some stochastic problems in real engineering [26-31]. In Model (7), $k_{i}(i=1,2,3)$ represents the feedback profit coefficient, which is also stochastic in a dynamic economic market. Unfortunately, there is very little literature related to the randomness of feedback profit coefficients. In fact, the randomness has the Markov property, for the feedback coefficient for the next moment is only related to that of the current moment. Therefore, in this paper, the author considers a Markovian jumping delayed impulsive reaction-diffusion financial system, which may be completed in three steps.

Step 1. Construct Markovian jumping delayed feedback financial system:

$$
\left\{\begin{array}{l}
\dot{x}=z+(y-a) x+k_{1}(r(t))\left(x-x\left(t-\tau_{1}(t)\right)\right) \\
\dot{y}=1-b y-x^{2}+k_{2}(r(t))\left(y-y\left(t-\tau_{2}(t)\right)\right) \\
\dot{z}=-x-c z+k_{3}(r(t))\left(z-z\left(t-\tau_{3}(t)\right)\right)
\end{array}\right.
$$


where $\left(\Omega_{*}, \mathcal{F}, \mathbb{P}\right)$ is the complete probability space defined in [32]. To avoid repetition in the narrative of the article, the author will not repeat it, and denotes $p_{i j}^{\delta}=\mathbb{P}(r(t+\delta)=j \mid r(t)=i)$.

Remark 7. In the definition of the transition probability, the quantity $1+\gamma_{i j} \delta$ is always well defined. Here is a brief explanation. For example, $0 \leqslant p_{i j}^{\delta} \leqslant 1, \sum_{j \in S} p_{i j}^{\delta}=1, \lim _{\delta \rightarrow 0} p_{i j}^{\delta}=0$ when $j \neq i$, and $\lim _{\delta \rightarrow 0} p_{i j}^{\delta}=1$ when $j=i$.

Step 2. Equilibrium point $Q_{1}$ of System (8) is translated into the null solution of the following system:

$$
\left\{\begin{array}{l}
\dot{X}_{1}=\frac{1}{c} X_{1}+\theta X_{2}+X_{3}+X_{1} X_{2}+k_{1}(r(t))\left(X_{1}-X_{1}\left(t-\tau_{1}(t)\right)\right) \\
\dot{X}_{2}=-2 \theta X_{1}-b X_{2}-X_{1}^{2}+k_{2}(r(t))\left(X_{2}-X_{2}\left(t-\tau_{2}(t)\right)\right) \\
\dot{X}_{3}=-X_{1}-c X_{3}+k_{3}(r(t))\left(X_{3}-X_{3}\left(t-\tau_{3}(t)\right)\right)
\end{array}\right.
$$

where $X_{i}(i=1,2,3)$ is defined in (2). Indeed, obviously, $Q_{1}$ is an equilibrium point of System (8), which corresponds to the equilibrium point $o(0,0,0)$ of System (9).

Step 3. Set up the reaction-diffusion model with impulse:

$$
\left\{\begin{array}{l}
\frac{\partial u}{\partial t}=D \Delta u-A u(t, x)+f(u(t, x))+K(r(t))(u-u(t-\tau(t), x)), \quad t \geqslant t_{0}=0, t \neq t_{k}, k \in \mathbb{Z}^{+} \\
u\left(t_{k}^{+}, x\right)=u\left(t_{k}, x\right)=B_{k} u\left(t_{k}^{-}, x\right), \quad t=t_{k}, k \in \mathbb{Z}^{+} \triangleq\{1,2, \cdots\} \\
u(s, x)=\xi(s, x), \quad(s, x) \in[-\tau, 0] \times \Omega \\
\frac{\partial u_{i}}{\partial v}=0, \quad x \in \partial \Omega, t \geqslant 0, i=1,2,3
\end{array}\right.
$$

where $\mathbb{Z}^{+} \triangleq\{1,2, \cdots\}$ represents $\mathbb{Z}^{+}=\{1,2, \cdots\}$. The state variable $\left.u(t-\tau(t), x)\right)=\left(u_{1}(t-\right.$ $\left.\left.\left.\left.\left.\tau_{1}(t), x\right)\right), u_{2}\left(t-\tau_{2}(t), x\right)\right), u_{3}\left(t-\tau_{3}(t), x\right)\right)\right)^{T}$, and $\Omega$ is a bounded subset of $R^{3}$. In addition, $\frac{\partial u_{i}}{\partial v}=$ $\left(\frac{\partial u_{i}}{\partial x_{1}}, \frac{\partial u_{i}}{\partial x_{2}}, \frac{\partial u_{i}}{\partial x_{3}}\right)^{T}$ represents the outward normal derivative on $\partial \Omega$, and the diffusion coefficient matrix $D=\operatorname{diag}\left(d_{1}, d_{2}, d_{3}\right)$ is a positive definite diagonal matrix in $R^{3 \times 3} . B_{k}$ and $\mathcal{N}$ are matrices in $R^{3 \times 3}$. $A$ is defined in (5), and:

$$
f(u)=\left(\begin{array}{c}
u_{1} u_{2} \\
-u_{1}^{2} \\
0
\end{array}\right), \quad K(r(t))=\left(\begin{array}{ccc}
k_{1}(r(t)) & 0 & 0 \\
0 & k_{2}(r(t)) & 0 \\
0 & 0 & k_{3}(r(t))
\end{array}\right) .
$$

Remark 8. The partially inaccessible transition rates model is more applicable for the real needs of complex financial market realities. Below, the global stability of the Markovian jumping delayed feedback financial system with partially unknown transition rates will be investigated.

For convenience, some fixed symbols and simple notations are introduced in this paper. For example, matrix $\mathfrak{A}>0, \mathfrak{A} \geqslant 0, \mathfrak{A} \geqslant \mathfrak{B}, \mathfrak{A}>\mathfrak{B}, L_{\mathcal{F}_{0}}^{2}\left([-\tau, 0] \times \Omega ; R^{3}\right)$ can be referred to the literature $[14,33]$ so that the author can avoid the high repetition rate of the article's narrative. In addition, denote by $\lambda_{1}$ the lowest positive eigenvalue of the Neumann boundary problem (see, e.g., [34]). To avoid repetition in the narrative of the article, the author adopts the symbols $S, S_{k n}^{r}, S_{u n}^{r}$, similarly to those of [32]. Denote $\widetilde{\alpha}_{r} \geqslant \max _{j \in S_{u n}^{r}}\left|\gamma_{r j}\right|$.

In this paper, the author will accomplish the following two main targets:

$\star$ Deriving the globally exponential stability criterion for the null solution of the impulsive system (6);

$\star$ Deducing the globally exponential stability criterion for the null solution of the impulsive system (10). 
2. Globally Exponential Stability of $Q_{1}$ with the Positive Interest Rate for the Reaction-Diffusion Model

Theorem 1. Suppose that $\dot{\tau}(t) \leqslant \tau_{0}<1$, and matrices $P_{r}=\operatorname{diag}\left(p_{r 1}, p_{r 2}, p_{r 3}\right)>0(r \in S)$ with $p_{r 1}=p_{r 2}$, satisfying:

$$
B_{k}^{T} P_{r} B_{k} \leqslant P_{i}, \quad \forall r, i \in S
$$

and for all $r \in S$,

$$
2 \lambda_{1} P_{r} D+P_{r} A+A^{T} P_{r}-\alpha P_{r}-\sum_{j \in S_{k n}^{r}}\left|\gamma_{r j}\right| P_{j}-\widetilde{\alpha}_{r} \sum_{j \in S_{u n}^{r}} P_{j}-2 P_{r} K_{r}-\left(\lambda_{\max } P_{r} K_{r}\right) I-\frac{\max _{r \in S}\left(\lambda_{\max } P_{r} K_{r}\right) e^{\alpha \tau}}{1-\tau_{0}} I>0,
$$

then the following two conclusions hold simultaneously:

(a) The null solution of System (10) is stochastically globally exponential stable in the mean square;

(b) The equilibrium point $Q_{1}\left(\sqrt{\theta}, \frac{1+a c}{c}, \frac{-\sqrt{\theta}}{c}\right)$ of the following financial system is stochastically globally exponential stability in the mean square:

$$
\left\{\begin{aligned}
& \frac{\partial u_{1}}{\partial t}=d_{1} \Delta u_{1}+\frac{1}{c}\left(u_{1}-\sqrt{\theta}\right)+\theta\left(u_{2}-\frac{1+a c}{c}\right)+\left(u_{3}-\frac{-\sqrt{\theta}}{c}\right) \\
&+\left(u_{1}-\sqrt{\theta}\right)\left(u_{2}-\frac{1+a c}{c}\right)+k_{1}(r(t))\left(u_{1}-u_{1}(t-\tau(t), x)\right), \quad t \geqslant 0, t \neq t_{k}, \\
& \quad+k_{2}(r(t))\left(u_{2}-u_{2}(t-\tau(t), x)\right), \quad t \geqslant 0, t \neq t_{k}, \\
& \frac{\partial u_{2}}{\partial t}= d_{2} \Delta u_{2}-2 \theta\left(u_{1}-\sqrt{\theta}\right)-b\left(u_{2}-\frac{1+a c}{c}\right)-\left(u_{1}-\sqrt{\theta}\right)^{2} \\
& \frac{\partial u_{3}}{\partial t}=d_{3} \Delta u_{3}-\left(u_{1}-\sqrt{\theta}\right)-c\left(u_{3}-\frac{-\sqrt{\theta}}{c}\right)+k_{3}(r(t))\left(u_{3}-u_{3}(t-\tau(t), x)\right), \quad t \geqslant 0, t \neq t_{k}, \\
& u\left(t_{k}^{+}, x\right)-Q_{1}=B_{k}\left[u\left(t_{k}^{-}, x\right)-Q_{1}\right], \quad t=t_{k}, k=1,2, \cdots \\
& u(s, x)=\xi(s, x)+Q_{1}, \quad(s, x) \in[-\tau, 0] \times \Omega, \\
& \frac{\partial u_{i}}{\partial v}=0, \quad x \in \partial \Omega, t \geqslant 0, i=1,2,3 .
\end{aligned}\right.
$$

Proof. Assume that $u$ is a solution of (10). I claim that:

$$
\int_{\Omega} u^{T} P_{r} D \Delta u d x \leqslant-\lambda_{1} \int_{\Omega} u^{T} P_{r} D u d x
$$

where $u_{i}(t, x) \in H_{0}^{1}(\Omega)$ for all $i \in\{1,2,3\}$ and $t \in[0,+\infty)$.

Indeed, one can conclude from the elliptic operators theory on the Laplacian eigenvalue [35] that $-\Delta$ is a self-adjoint compact inverse operator under the Neumann zero boundary value. Next, similarly to the proof of [34] (Lemma 2.1), one can see, for any $u_{i}(x) \in H_{0}^{1}(\Omega), u_{i}(x)=\sum_{k=0}^{\infty} c_{k} \vartheta(x)$ and:

$$
\int_{\Omega}\left|\nabla u_{i}(x)\right|^{2} d x \geqslant \lambda_{1} \int_{\Omega} u_{i}^{2}(x) d x
$$

Let $u$ be a solution of the system (10), then one can conclude from (16), the Gauss formula, and the Neumann zero boundary condition that:

$$
\begin{aligned}
& \int_{\Omega} u^{T} P_{r} D \Delta u d x \\
= & -\sum_{k=1}^{3} \sum_{j=1}^{3} \int_{\Omega} p_{r j} d_{j}\left(\frac{\partial u_{j}}{\partial x_{k}}\right)^{2} d x \\
\leqslant & -\lambda_{1} \sum_{j=1}^{3} \int_{\Omega} u_{j} p_{r j} d_{j} u_{j} d x=-\lambda_{1} \int_{\Omega} u^{T} P_{r} D u d x,
\end{aligned}
$$


which proves the claim (15), where $D=\operatorname{diag}\left(d_{1}, d_{2}, d_{3}\right), P_{r}=\left(p_{r 1}, p_{r 2}, p_{r 3}\right)$.

Consider the Lyapunov-Krasovskii functional: for any mode $r \in S, V_{r}(t)=V_{r 1}(t)+V_{r 2}(t)$ with:

$$
V_{r 1}(t)=e^{\alpha t} \int_{\Omega} u^{T} P_{r} u d x
$$

and:

$$
V_{r 2}(t)=\frac{c_{1} e^{\alpha \tau}}{1-\tau_{0}} \int_{\Omega} \int_{t-\tau(t)}^{t} e^{\alpha s} u^{T}(s, x) u(s, x) d s d x,
$$

where the constant scalar $c_{1}=\max _{r \in S}\left(\lambda_{\max } P_{r} K_{r}\right)$.

From the definition of $\widetilde{\alpha}_{r}$, it is clear that:

$$
u^{T} \sum_{j \in S} \gamma_{r j} P_{j} u \leqslant u^{T} \sum_{j \in S_{k n}^{r}}\left|\gamma_{r j}\right| P_{j} u+u^{T} \widetilde{\alpha}_{r} \sum_{j \in S_{u n}^{r}} P_{j} u .
$$

Let $\mathcal{L}$ be the weak infinitesimal operator (see, e.g., [14]) such that:

$$
\begin{aligned}
\mathcal{L} V_{r 1} \leqslant & e^{\alpha t} \int_{\Omega}\left[u^{T}\left(\alpha P_{r}+\sum_{j \in S} \gamma_{r j} P_{j}-2 \lambda_{1} P_{r} D-\left(P_{r} A+A^{T} P_{r}\right)\right) u\right. \\
& \left.+2 u^{T} P_{r} f(u)+2 u^{T} P_{r} K_{r}(u-u(t-\tau(t), x))\right] d x \\
\leqslant & e^{\alpha t} \int_{\Omega}\left[u ^ { T } \left(\alpha P_{r}+\sum_{j \in S_{k n}^{r}}\left|\gamma_{r j}\right| P_{j}+\widetilde{\alpha}_{r} \sum_{j \in S_{u n}^{r}} P_{j}-2 \lambda_{1} P_{r} D-\left(P_{r} A+A^{T} P_{r}\right)+2 P_{r} K_{r}\right.\right. \\
& \left.\left.\left.+\left(\lambda_{\max } P_{r} K_{r}\right) I\right) u+\max _{r \in S}\left(\lambda_{\max } P_{r} K_{r}\right) u^{T}(t-\tau(t), x) u(t-\tau(t), x)\right)\right] d x
\end{aligned}
$$

and:

$$
\begin{aligned}
\mathcal{L} V_{r 2}(t) & =\frac{c_{1} e^{\alpha \tau}}{1-\tau_{0}} \int_{\Omega} e^{\alpha t} u^{T}(t, x) u(t, x) d x-\frac{c_{1} e^{\alpha \tau}}{1-\tau_{0}}(1-\dot{\tau}(t)) \int_{\Omega} e^{\alpha(t-\tau(t))} u^{T}(t-\tau(t), x) u(t-\tau(t), x) d x \\
& \leqslant \frac{c_{1} e^{\alpha \tau}}{1-\tau_{0}} \int_{\Omega} e^{\alpha t} u^{T}(t, x) u(t, x) d x-c_{1} \int_{\Omega} e^{\alpha t} u^{T}(t-\tau(t), x) u(t-\tau(t), x) d x
\end{aligned}
$$

Combining (13), (19), and (20) results in:

$$
\begin{aligned}
\mathcal{L} V_{r}(t) \leqslant e^{\alpha t} \int_{\Omega} u^{T}\left(\alpha P_{r}+\sum_{j \in S_{k n}^{r}}\left|\gamma_{r j}\right| P_{j}+\widetilde{\alpha}_{r} \sum_{j \in S_{u n}^{r}} P_{j}-2 \lambda_{1} P_{r} D-\left(P_{r} A+A^{T} P_{r}\right)+2 P_{r} K_{r}\right. \\
\left.+\left(\lambda_{\max } P_{r} K_{r}\right) I+\frac{c_{1} e^{\alpha \tau}}{1-\tau_{0}} I\right) u d x \leqslant 0, \quad \forall t \in \bigcup_{k=0}^{\infty}\left[t_{k}, t_{k+1}\right),
\end{aligned}
$$

which means

$$
\mathbb{E} V(t, r(t)) \leqslant \mathbb{E} V\left(t_{k}, r\left(t_{k}\right)\right), \quad \forall t \in\left[t_{k}, t_{k+1}\right), k=0,1,2 \cdots .
$$

On the other hand, for any modes $r, i \in S$,

$$
\begin{aligned}
V\left(t_{k}, r\right)-V\left(t_{k}^{-}, i\right) \leqslant & e^{\alpha t}\left[\int_{\Omega} u^{T}\left(t_{k}, x\right) P_{r} u\left(t_{k}, x\right) d x-\int_{\Omega} u^{T}\left(t_{k}^{-}, x\right) P_{i} u\left(t_{k}^{-}, x\right) d x\right] \\
& +\frac{c_{1} e^{\alpha \tau}}{1-\tau_{0}} \int_{\Omega}\left[\int_{t_{k}-\tau\left(t_{k}\right)}^{t_{k}} e^{\alpha s} u^{T}(s, x) u(s, x) d s-\int_{t_{k}^{-}-\tau\left(t_{k}^{-}\right)}^{t_{k}^{-}} e^{\alpha s} u^{T}(s, x) u(s, x) d s\right] d x \\
\leqslant & e^{\alpha t}\left[\int_{\Omega} u^{T}\left(t_{k}^{-}, x\right)\left(B_{k}^{T} P_{r} B_{k}-P_{i}\right) u\left(t_{k}^{-}, x\right) d x\right] \leqslant 0 .
\end{aligned}
$$


Combining (21) and (22) reaches:

$\mathbb{E} V(t, r(t)) \leqslant \mathbb{E} V\left(t_{k}, r\left(t_{k}\right)\right) \leqslant \mathbb{E} V\left(t_{k}^{-}, r\left(t_{k}^{-}\right)\right) \leqslant \mathbb{E} V\left(t_{k-1}, r\left(t_{k-1}\right)\right) \leqslant \cdots \leqslant \mathbb{E} V\left(t_{0}, r\left(t_{0}\right)\right), \quad \forall t \geqslant t_{0}=0$.

Moreover,

$$
\begin{aligned}
\min _{r \in S}\left(\lambda_{\min } P_{r}\right) e^{\alpha t} \mathbb{E}\|u\|^{2} & \leqslant \mathbb{E} V(t, r(t)) \leqslant \mathbb{E} V\left(t_{0}, r\left(t_{0}\right)\right) \\
& =\mathbb{E}\left(e^{\alpha t_{0}} \int_{\Omega} \xi^{T}(0, x) P\left(r\left(t_{0}\right)\right) \xi(0, x) d x+\frac{c_{1} e^{\alpha \tau}}{1-\tau_{0}} \int_{\Omega} \int_{0-\tau(0)}^{0} e^{\alpha s} \xi^{T}(0, x) \xi(0, x) d s d x\right) \\
& \leqslant\left(\left(\lambda_{\max } P(r(0))\right)+\frac{\tau c_{1} e^{\alpha \tau}}{1-\tau_{0}}\right) \sup _{-\tau \leqslant s \leqslant 0} \mathbb{E}\|\xi(s)\|^{2}, \quad \forall t \geqslant 0,
\end{aligned}
$$

which implies:

$$
\mathbb{E}\|u\|^{2} \leqslant c_{2} \sup _{-\tau \leqslant s \leqslant 0} \mathbb{E}\|\xi(s)\|^{2} e^{-\alpha t}, \quad \forall t \geqslant 0,
$$

which means the completion of the proof of Theorem 1 , where $c_{2}$ is a suitable positive constant scalar.

\section{Impulse Control on the Financial System without Time-Delays}

For simplicity, in this section, the author denotes the norm $\|\cdot\|$ as follows,

$$
\|Y\|^{2}=Y^{T} Y, \quad \text { where } Y \in R^{3} .
$$

Theorem 2. Assume that there exist positive scalars $q_{k}, \varsigma, \lambda>0$ with $\varsigma-\lambda \geqslant \lambda_{\max }\left(-A^{T}-A\right)$ such that:

$$
\lambda_{\max } B_{k}^{T} B_{k} \leqslant q_{k}<e^{-(\varsigma+\lambda)\left(t_{k+1}-t_{k}\right)}, \quad k \in \mathbb{Z}^{+},
$$

then the following two conclusions hold simultaneously:

(a) the null solution of the system (6) is globally exponential stable with the convergence rate $\frac{\lambda}{2}$;

(b) the equilibrium point $Q_{1}$ with positive interest rate $\sqrt{\theta}$ for the following system is globally exponential stable with the convergence rate $\frac{\lambda}{2}$ :

$$
\left\{\begin{array}{l}
\dot{x}=z+(y-a) x, \quad t \geqslant 0, t \notin\left\{t_{k}, k \in \mathbb{Z}^{+}\right\}, \\
\dot{y}=1-b y-x^{2}, \quad t \geqslant 0, t \notin\left\{t_{k}, k \in \mathbb{Z}^{+}\right\}, \\
\dot{z}=-x-c z, \quad t \geqslant 0, t \notin\left\{t_{k}, k \in \mathbb{Z}^{+}\right\}, \\
\left(x\left(t_{k}^{+}\right)-\sqrt{\theta}, y\left(t_{k}^{+}\right)-\frac{1+a c}{c}, z\left(t_{k}^{+}\right)+\frac{\sqrt{\theta}}{c}\right)^{T} \\
=B_{k}\left(x\left(t_{k}^{-}\right)-\sqrt{\theta}, y\left(t_{k}^{-}\right)-\frac{1+a c}{c}, z\left(t_{k}^{-}\right)+\frac{\sqrt{\theta}}{c}\right)^{T}, t=t_{k}, k \in \mathbb{Z}^{+}, \\
\left(x\left(t_{0}\right)-\sqrt{\theta}, y\left(t_{0}\right)-\frac{1+a c}{c}, z\left(t_{0}\right)+\frac{\sqrt{\theta}}{c}\right)^{T}=X_{0} .
\end{array}\right.
$$

Proof. Let $X$ be a solution of impulsive system (6); the author considers the following norm:

$$
\|X\|^{2}=X^{T} X
$$

Firstly, I claim that there exists positive scalar $\mathfrak{C} \geqslant e^{(\varsigma+\lambda)\left(t_{k}-t_{k-1}\right)}>1$ such that:

$$
X^{T}(t) X(t) \leqslant \mathfrak{C}\left\|X_{0}\right\|^{2} e^{-\lambda\left(t-t_{0}\right)}, \quad t \in\left[t_{k-1}, t_{k}\right), k \in \mathbb{Z}^{+} .
$$

Indeed, from the condition (26), one sees: 


$$
0<q_{k-1} \leqslant e^{-(\varsigma+\lambda)\left(t_{k}-t_{k-1}\right)}<1 .
$$

Hence,

$$
0<q_{k-1}<e^{(\varsigma+\lambda)\left(t_{k}-t_{k-1}\right)} \leqslant \mathfrak{C}
$$

which implies:

$$
\begin{aligned}
e^{(\varsigma+\lambda)\left(t_{k}-t_{k-1}\right)} \leqslant \mathfrak{C} & \Rightarrow 0<\left\|X_{0}\right\|^{2} \leqslant\left\|X_{0}\right\|^{2} e^{\varsigma\left(t_{k}-t_{k-1}\right)} \leqslant \mathfrak{C}\left\|X_{0}\right\|^{2} e^{-\lambda\left(t_{k}-t_{k-1}\right)} \\
& \Rightarrow 1=e^{0}<e^{\varsigma\left(t_{k}-t_{k-1}\right)} \leqslant \mathfrak{C} e^{-\lambda\left(t_{k}-t_{k-1}\right)}, \quad k \in \mathbb{Z}^{+} .
\end{aligned}
$$

To prove (29), I firstly prove:

$$
\|X\|^{2} \leqslant \mathfrak{C}\left\|X_{0}\right\|^{2} e^{-\lambda\left(t-t_{0}\right)}, \quad t \in\left[t_{0}, t_{1}\right) .
$$

Due to $e^{-\lambda\left(t_{1}-t_{0}\right)} \leqslant e^{-\lambda\left(t-t_{0}\right)}$ for all $t \in\left[t_{0}, t_{1}\right)$, I only need to prove:

$$
\|X(t)\|^{2} \leqslant \mathfrak{C}\left\|X_{0}\right\|^{2} e^{\left(t_{0}-t_{1}\right) \lambda}, \quad t \in\left[t_{0}, t_{1}\right) .
$$

I consider reduction to absurdity similarly to that of the proof for [36] (Theorem 1). Obviously, (34) holds at $t=t_{0}$, as a result of (32) and $\left\|X\left(t_{0}\right)\right\|=\left\|X_{0}\right\|$. Moreover,

$$
\left\|X\left(t_{0}\right)\right\|^{2}=1 \cdot\left\|X_{0}\right\|^{2}<\mathfrak{C}\left\|X_{0}\right\|^{2} e^{-\lambda\left(t_{1}-t_{0}\right)} .
$$

If (34) does not hold, there is a scalar $\iota$ with $t_{0}<\iota<t_{1}$ such that:

$$
\|X(\iota)\|^{2}>\mathfrak{C}\left\|X_{0}\right\|^{2} e^{-\lambda\left(t_{1}-t_{0}\right)} \geqslant\left\|X_{0}\right\|^{2} e^{\varsigma\left(t_{1}-t_{0}\right)}>\left\|X_{0}\right\|^{2} .
$$

This deduces that there is a positive constant $t_{*}$ with $t_{0}<t_{*}<\iota$ such that:

$$
\left\|X\left(t_{*}\right)\right\|^{2}=\mathfrak{C}\left\|X_{0}\right\|^{2} e^{-\lambda\left(t_{1}-t_{0}\right)}, \quad \text { and } \quad\|X(t)\|^{2} \leqslant \mathfrak{C}\left\|X_{0}\right\|^{2} e^{-\lambda\left(t_{1}-t_{0}\right)}, \forall t \in\left[t_{0}, t_{*}\right],
$$

and there is positive constant $t_{* *}$ with $t_{0} \leqslant t_{* *}<t_{*}$ such that for $t \in\left[t_{* *}, t_{*}\right]$,

$$
\left\|X\left(t_{* *}\right)\right\|^{2}=\left\|X_{0}\right\|^{2},
$$

which implies together with (32) that:

$$
\left\|X_{0}\right\|^{2} \leqslant \mathfrak{C}\left\|X_{0}\right\|^{2} e^{-\lambda\left(t_{1}-t_{0}\right)} \leqslant \mathfrak{C}\|X(t)\|^{2} e^{-\lambda\left(t_{1}-t_{0}\right)}, t \in\left[t_{* *}, t_{*}\right] .
$$

Let $D^{+}$be the upper right derivative (Dini derivative) such that for $t \in\left[t_{*^{*}}, t_{*}\right] \subset\left[t_{0}, t_{1}\right)$,

$$
D^{+}\left(X^{T} X\right) \leqslant \lambda_{\max }\left(-A^{T}-A\right)\|X\|^{2} \leqslant(\varsigma-\lambda) X^{T} X,
$$

and hence,

$$
\begin{aligned}
X^{T}\left(t_{*}\right) X\left(t_{*}\right) & \leqslant X^{T}\left(t_{* *}\right) X\left(t_{* *}\right) e^{(\varsigma-\lambda)\left(t_{*}-t_{* *}\right)} \\
& <\left\|X_{0}\right\|^{2} e^{(\varsigma+\lambda)\left(t_{1}-t_{0}\right)} e^{-\lambda\left(t_{1}-t_{0}\right)} \leqslant \mathfrak{C}\left\|X_{0}\right\|^{2} e^{-\lambda\left(t_{1}-t_{0}\right)}=\left\|X\left(t_{*}\right)\right\|^{2} .
\end{aligned}
$$

This is a contradiction, and then, (29) holds for $k=1$.

Assume that (29) is fulfilled for all $1 \leqslant k \leqslant m$ with $m \in \mathbb{Z}^{+}$. That is,

$$
\|X(t)\|^{2} \leqslant \mathfrak{C}\left\|X_{0}\right\|^{2} e^{-\lambda\left(t-t_{0}\right)}, t \in\left[t_{k-1}, t_{k}\right), k \in\{1,2, \cdots, m\} .
$$


By (39), I shall show that (29) is fulfilled for $k=m+1$, i.e.,

$$
X^{T}(t) X(t) \leqslant \mathfrak{C}\left\|X_{0}\right\|^{2} e^{-\lambda\left(t-t_{0}\right)}, t \in\left[t_{m}, t_{m+1}\right) .
$$

In fact, to prove (40), I only need to prove:

$$
X^{T}(t) X(t) \leqslant \mathfrak{C}\left\|X_{0}\right\|^{2} e^{-\lambda\left(t_{m+1}-t_{0}\right)}, t \in\left[t_{m}, t_{m+1}\right) .
$$

First, I claim:

$$
X^{T}\left(t_{m}\right) X\left(t_{m}\right)<\mathfrak{C}\left\|X_{0}\right\|^{2} e^{-\lambda\left(t_{m+1}-t_{0}\right)}
$$

Indeed,

$$
\begin{aligned}
X^{T}\left(t_{m}\right) X\left(t_{m}\right) & \leqslant q_{m} \mathfrak{C}\left\|X_{0}\right\|^{2} e^{-\lambda\left(t_{m}-t_{0}\right)} \\
& <e^{-\lambda\left(t_{m+1}-t_{m}\right)} \mathfrak{C}\left\|X_{0}\right\|^{2} e^{-\lambda\left(t_{m}-t_{0}\right)}=\mathfrak{C}\left\|X_{0}\right\|^{2} e^{-\lambda\left(t_{m+1}-t_{0}\right)} .
\end{aligned}
$$

If (41) is not fulfilled, there is $t^{*} \in\left(t_{m}, t_{m+1}\right)$ with $t^{*}=\inf _{t \in\left[t_{m}, t_{m+1}\right)}\left\{t: X^{T}(t) X(t)>\right.$ $\left.\mathfrak{C}\left\|X_{0}\right\|^{2} e^{-\lambda\left(t_{m+1}-t_{0}\right)}\right\}$ such that:

$$
\left\|X\left(t^{*}\right)\right\|^{2}=\mathfrak{C}\left\|X_{0}\right\|^{2} e^{-\lambda\left(t_{m+1}-t_{0}\right)}, \text { and } X^{T}(t) X(t) \leqslant \mathfrak{C}\left\|X_{0}\right\|^{2} e^{-\lambda\left(t_{m+1}-t_{0}\right)}, t \in\left[t_{m}, t^{*}\right) .
$$

It follows from (42) that there exists $t^{* *} \in\left[t_{m}, t^{*}\right)$ satisfying:

$$
\left\|X\left(t^{* *}\right)\right\|^{2}=q_{m} \mathfrak{C}\left\|X_{0}\right\|^{2} e^{-\lambda\left(t_{m}-t_{0}\right)} .
$$

Due to $D^{+}\|X(t)\|^{2} \leqslant(\varsigma-\lambda)\|X(t)\|^{2}$ on $\left[t^{* *}, t^{*}\right]$, one can similarly get:

$$
X^{T}\left(t^{*}\right) X\left(t^{*}\right) \leqslant\left\|X\left(t^{* *}\right)\right\|^{2} e^{(\varsigma-\lambda)\left(t^{*}-t^{* *}\right)}<\mathfrak{C}\left\|X_{0}\right\|^{2} e^{-\lambda\left(t_{m+1}-t_{0}\right)}=\left\|X\left(t^{*}\right)\right\|^{2} .
$$

This is a contradiction. Thus, (41) holds, and hence, (40) holds. Mathematical induction yields that (29) holds for each $k \in \mathbb{Z}^{+}$. The condition (26) implies:

$$
X^{T}\left(t_{k}\right) X\left(t_{k}\right)<\left\|X\left(t_{k}^{-}\right)\right\|^{2},
$$

which together with (29) deduces:

$$
\|X\|^{2} \leqslant \mathfrak{C}\left\|X_{0}\right\|^{2} e^{-\lambda\left(t-t_{0}\right)}, \quad t \in\left[t_{k-1}, t_{k}\right], \text { for all } k \in \mathbb{Z}^{+} .
$$

This has actually completed the proof of Theorem 2.

Remark 9. Theorem 2 presents a simple and feasible stability criterion on the equilibrium point $Q_{1}$ with the positive interest rate for the complex financial system (27), whose feasibility can be verified in the numerical example below.

\section{Numerical Example}

Example 1. In System (10) or (14), let $a=0.9, b=0.2, c=0.2463$, and then:

$$
A=\left(\begin{array}{ccc}
-4.0601 & -0.0893 & -1.0000 \\
0.1787 & 0.2000 & 0 \\
1.0000 & 0 & 0.2463
\end{array}\right) \text {. }
$$


Let $S=\{1,2,3\}$ and the transition rates matrix $\Pi$, feedback gain coefficient matrices, diffusion coefficient matrix, and impulse coefficient matrices be given as follows:

$$
\begin{aligned}
& \Pi=\left(\begin{array}{ccc}
-0.026 & ? & ? \\
? & -0.023 & ? \\
0.016 & 0.013 & -0.029
\end{array}\right), \quad K_{1}=\left(\begin{array}{ccc}
0.011 & 0 & 0 \\
0 & 0.015 & 0 \\
0 & 0 & 0.012
\end{array}\right) \\
& K_{2}=\left(\begin{array}{ccc}
0.012 & 0 & 0 \\
0 & 0.016 & 0 \\
0 & 0 & 0.013
\end{array}\right), \quad K_{3}=\left(\begin{array}{ccc}
0.01101 & 0 & 0 \\
0 & 0.0151 & 0 \\
0 & 0 & 0.011
\end{array}\right) \text {. } \\
& D=\left(\begin{array}{ccc}
1.9165 & 0 & 0 \\
0 & 1.9161 & 0 \\
0 & 0 & 1.9163
\end{array}\right), B_{k}=\left(\begin{array}{ccc}
\frac{1}{18 k^{3}} & 0 & 0 \\
0 & \frac{1}{16 k^{5}} & 0 \\
0 & 0 & 0.0010
\end{array}\right), k=1,2, \cdots
\end{aligned}
$$

Take $\widetilde{\alpha}_{1}=0.026, \widetilde{\alpha}_{2}=0.023$, and let $\Omega$ be a unit ball in $R^{3}$, then $\lambda_{1}=2.1762$. Let $\tau=0.5$, $\tau_{0}=0.3, \alpha=0.0012$, I can employ MATLAB software to solve the inequalities (3.2), obtaining the following feasible solutions:

$$
\begin{gathered}
P_{1}=\left(\begin{array}{ccc}
1.0101 & 0 & 0 \\
0 & 1.0101 & 0 \\
0 & 0 & 1.0780
\end{array}\right), P_{2}=\left(\begin{array}{ccc}
1.0021 & 0 & 0 \\
0 & 1.0021 & 0 \\
0 & 0 & 1.0130
\end{array}\right), \\
P_{3}=\left(\begin{array}{ccc}
1.0132 & 0 & 0 \\
0 & 1.0132 & 0 \\
0 & 0 & 1.0011
\end{array}\right),
\end{gathered}
$$

which guarantee that the conditions (12) and (13) hold. Thus, Theorem 1 yields that $Q_{1}$ of (14) is stochastically globally exponential stable in the mean square.

Remark 10. In Example 1, $a=0.9, b=0.2, c=0.2463$ derive that $c-b-a b c=0.0020>0$ and $c+a-$ $\frac{1}{b}=-3.8537<0$. Remark 1 demonstrates that chaos appears in the financial system (1) if $c-b-a b c>0$ and $c+a-\frac{1}{b}<0$. However, with the help of impulsive control and regional control, one sees that the equilibrium point $Q_{1}$ for the delayed feedback financial system (14) is stochastically globally exponential stable. Particularly, the interest rate is $0.0893>0$ when the financial system reaches stability, which means better economic significance (see Table 1).

Table 1. Comparisons of System (1) and System (14) under the same data $a=0.9, b=0.2, c=0.25$.

\begin{tabular}{cccc}
\hline & System (1) & System (10) & Positive or Negative \\
\hline$c-b-a b c$ & 0.0050 & 0.0050 & $>0$ \\
$c+a-\frac{1}{b}$ & -3.8500 & -3.8500 & $<0$ \\
system state & chaos & global stability & \\
interest rate of $Q_{1}$ & $8.93 \%$ & $8.93 \%$ & $>0$ \\
\hline
\end{tabular}

Remark 11. Table 1 illustrates that impulsive control and regional control stabilize the chaotic financial system. When the financial system reaches stability, the interest rate is the positive number $8.93 \%$.

Example 2. In the system (6) or (27), let $a=0.9, b=0.2, c=0.2463$, and one gets":

$$
A=\left(\begin{array}{ccc}
-4.0601 & -0.0893 & -1.0000 \\
0.1787 & 0.2000 & 0 \\
1.0000 & 0 & 0.2463
\end{array}\right)
$$


In addition, let $q_{k} \equiv 0.6, \varsigma=9.3, \lambda=0.7, t_{k+1}-t_{k} \equiv 0.05$,

$$
B_{k}=\left(\begin{array}{ccc}
\frac{1}{18 k^{3}} & 0 & 0 \\
0 & \frac{1}{16 k^{5}} & 0 \\
0 & 0 & 0.0010
\end{array}\right), k=1,2, \cdots
$$

Now, one can compute and obtain:

$$
\begin{gathered}
\lambda_{\max } B_{k}^{T} B_{k} \leqslant \lambda_{\max } B_{1}^{T} B_{1}<0.6=q_{k}, \\
\varsigma-\lambda=8.6 \geqslant 8.1211=\lambda_{\max }\left(-A^{T}-A\right), \\
q_{k}=0.6<0.6065=e^{-(\varsigma+\lambda)\left(t_{k+1}-t_{k}\right),}
\end{gathered}
$$

which implies that the condition (26) is satisfied. Hence, Theorem 2 yields that the equilibrium point $Q_{1}$ with positive interest rate $8.93 \%$ for the financial system (27) is globally exponential stable with convergence rate 0.35 .

Remark 12. Example 2 illustrates that the condition (26) is easily satisfied, which verifies the feasibility of Theorem 2. In fact, Theorem 2 is easier to verify than those of existing results ([9] (Theorems 1-2)).

Remark 13. The same data are proposed for Examples 1 and 2. This indicates that time delay really brings the essential difficulty in stability analysis of complex financial systems, for the regional control has to be adopted to deal with stability analysis of the delayed feedback financial system while the financial system without time delays can be stabilized directly by impulse control.

\section{Conclusions and Further Considerations}

In this paper, the author firstly employed impulsive control, regional control, the Lyapunov function, and variational methods to derive the stochastically globally exponential stability of the economic balance point with the positive interest rate for the Markovian jumping delayed feedback financial system with partially unknown transition rates. Besides, the mathematical induction method and the proof by contradiction were applied synthetically to derive the globally exponential stability of the equilibrium point with the positive interest rate for the impulsive financial system without time delays. The same data were proposed for Examples 1 and 2. This illustrates that time delay really brings the essential difficulty in stability analysis of a complex financial system, for the regional control has to be adopted to deal with the stability analysis of the delayed feedback financial system while the financial system without time delays can be stabilized directly by impulse control. In summary, if time delay is not considered, impulse control can make the financial system stable by adjusting the impulse quantity and impulse interval; if the time delay feedback factor is considered, the regional control method and impulse control method can stabilize the chaotic financial system by adjusting the diffusion coefficient and impulse.

In addition, it should be pointed out that the equilibrium point $Q_{1}$ with the positive interest rate brings more difficulties than the equilibrium point $Q_{0}(0,1 / b, 0)$ with zero interest rate, for the stability of $Q_{0}(0,1 / b, 0)$ can be obtained under some suitable data without impulse control, even in the delayed feedback financial system. However, the stability of $Q_{1}$ with the positive interest rate cannot be derived under any suitable data if impulse control is not applied. Theorem 2 presents a globally exponential stability criterion for the equilibrium point $Q_{1}$ with the positive interest rate under impulse control. The global stability of the equilibrium point $Q_{1}$ with the positive interest rate of the delayed feedback financial system may not be derived only under impulse control. Of course, I do not know whether the global stability of $Q_{1}$ for the delayed feedback financial system can be deduced only under impulse control. It is an interesting problem.

Under Lipschitz conditions ensuring the unique existence of the solution of the reaction-diffusion system for any given initial value, Ruofeng Rao, Shouming Zhong, and Zhilin Pu deduced the 
boundedness conclusion [25] (Theorem 3.3) and the stability criterion [25] (Theorem 3.4), in which the following formula was derived:

$$
\lambda_{1}\left(B\left(0, R_{0}\right)\right)=\left\{\begin{array}{l}
\frac{\beta_{0}^{2}\left(\frac{\pi^{m}}{m !}\right)^{\frac{2}{2 m}}}{\left[m e s\left(B\left(0, R_{0}\right)\right)\right]^{\frac{2}{2 m}}}=\frac{\beta_{0}^{2}\left(\frac{\pi^{\frac{n}{2}}}{\left(\frac{n}{2}\right) !}\right)^{\frac{2}{n}}}{\left[m e s\left(B\left(0, R_{0}\right)\right)\right]^{\frac{2}{n}}}, \quad n=2 m, \\
\frac{\beta_{0}^{2} \frac{\left[2(2 \pi)^{m}\right] \frac{2}{2 m+1}}{(2 m+1) ! !}}{\left[m e s\left(B\left(0, R_{0}\right)\right)\right]^{\frac{2}{2 m+1}}}=\frac{\beta_{0}^{2} \frac{\left[2(2 \pi)^{\frac{n-1}{2}}\right]^{\frac{2}{n}}}{n ! !}}{\left[\operatorname{mes}\left(B\left(0, R_{0}\right)\right)\right]^{\frac{2}{n}}}, \quad n=2 m+1 .
\end{array}\right.
$$

This has actually proven the following conclusion:

Theorem 3. If $f_{i}, \tilde{f}_{i}, \sigma_{i j}, \hat{\sigma}_{i j}$ are Lipschitz continuous with $f_{i}(0)=\tilde{f}_{i}(0)=\sigma_{i j}(0)=\hat{\sigma}_{i j}(0)=0$, then there must exist a series of spherical regions $B\left(0, R_{0}\right) \subset R^{n}$ with $R_{0}$ moderately small such that the following fuzzy system (47) is globally stochastically exponential stable in the $p^{\text {th }}$ moment.

$$
\left\{\begin{aligned}
d u_{i}(t, x)= & q_{i} d i v \nabla u_{i}(t, x) d t-\sum_{r=1}^{r_{*}} \varrho_{r}(\hat{\omega}(t))\left[a_{i r} u_{i}(t, x)-\sum_{j=1}^{n} b_{i j r} f_{j}\left(v_{j}(t, x)\right)-\sum_{j=1}^{n} c_{i j r} f_{j}\left(v_{j}(t-\tau(t), x)\right)\right. \\
& \left.-\sum_{j=1}^{n} h_{i j r} \int_{t-\rho(t)}^{t} f_{j}\left(v_{j}(s, x)\right) d s\right] d t+\sum_{j=1}^{n} \sigma_{i j}(t, u(t, x), v(t-\tau(t), x)) d w_{j}(t), \quad t \geqslant 0, x \in \mathrm{Y}, \\
d v_{i}(t, x)= & \tilde{q}_{i} d i v \nabla v_{i}(t, x) d t-\sum_{r=1}^{r_{*}} \varrho_{r}(\hat{\omega}(t))\left[\tilde{a}_{i r} v_{i}(t, x)-\sum_{j=1}^{n} \tilde{b}_{i j r} \tilde{f}_{j}\left(u_{j}(t, x)\right)-\sum_{j=1}^{n} \tilde{c}_{i j r} \tilde{f}_{j}\left(u_{j}(t-\hat{\tau}(t), x)\right)\right. \\
& \left.-\sum_{j=1}^{n} \tilde{h}_{i j r} \int_{t-\tilde{\rho}(t)}^{t} \tilde{f}_{j}\left(u_{j}(s, x)\right) d s\right] d t+\sum_{j=1}^{n} \hat{\sigma}_{i j}(t, v(t, x), u(t-\hat{\tau}(t), x),) d \tilde{w}_{j}(t), \quad t \geqslant 0, x \in \mathrm{Y}, \\
u_{i}(t, x)= & \zeta_{i}(t, x), v_{i}(t, x)=\omega_{i}(t, x), \quad \forall(s, x) \in[-\tau, 0] \times \mathrm{Y} \\
\partial_{v} u(t, x)= & =\partial_{v} v(t, x), \quad \forall(t, x) \in[0,+\infty] \times \partial \mathrm{Y} .
\end{aligned}\right.
$$

How to derive the concise criterion for the financial systems (10), similarly to that of Theorem 3 is an interesting problem.

Funding: The research is supported by the China National College Students Innovation and Entrepreneurship Training Program at Chengdu Normal University in 2018 (No. 201814389083), the Scientific Fund of the Education Department of Sichuan Province in China (No. 18ZA0082), Chengdu Normal University's teaching reform project (No. 2018JG38) in 2018, and Chengdu Normal University's Nurturing Projects in 2018 (No. CS18ZD08).

Conflicts of Interest: The author declares no conflict of interest.

\section{References}

1. Cheng, S. Complicated Science and Management. Article Collection of Beijing Xiangshan Conference; Science Press: Beijing, China, 1998; pp. 1-9. (In Chinese)

2. Huang, D.; Li, H. Theory and Method of Nonlinear Economics; Sichuan University Press: Chengdu, China, 1993.

3. Ma, J.; Chen, Y. Study for the bifurcation topological structure and the global complicated character of a kind of nonlinear finance system (I). Appl. Math. Mech. 2001, 11, 1240-1251. [CrossRef]

4. Ma, J.; Chen, Y. Study for the bifurcation topological structure and the global complicated character of a kind of nonlinear finance system (II). Appl. Math. Mech. 2001, 12, 1375-1382. [CrossRef]

5. Chen, W. Dynamics and control of a financial system with time-delayed feedbacks. Chaos Solitons Fractals 2008, 37, 1198-1207. [CrossRef]

6. Zhao, M.; Wang, J. $H_{\infty}$ control of a chaotic finance system in the presence of external disturbance and input time-delay. Appl. Math. Comput. 2014, 233, 320-327. [CrossRef]

7. Zhao, X.; Li, Z.; Li, S. Synchronization of a chaotic finance system. Appl. Math. Comput. 2011, 217, 6031-6039. [CrossRef] 
8. Chen, Y. Chaos control for a class of nonlinear finance systems model. Math. Pract. Theory 2019, 49, 18-26. (In Chinese)

9. Wang, Y. Impulsive Control of a Chaotic Financial System. In Proceedings of the 4th International Conference on Machinery, Materials and Computer (MACMC 2017), Xi'an, China, 27-29 November 2017; Volume 150, pp. 548-552.

10. Li, X.; Yang, X.; Huang, T. Persistence of delayed cooperative models: Impulsive control method. Appl. Math. Comput. 2019, 342, 130-146 [CrossRef]

11. Zhang, R. Bifurcation Analysis for a Kind of Nonlinear Finance System with Delayed Feedback and Its Application to Control of Chaos. J. Appl. Math. 2012. [CrossRef]

12. Yi, A.; Zi, A.; Rao, R.; Zhao, F.; Huang, H. Impulse Control of Financial System with Probabilistic Delay Feedback. Appl. Math. Mech. 2019. (In Chinese) [CrossRef]

13. Luo, M.; Liu, X.; Zhong, S. Synchronization of stochastic complex networks with discrete-time and distributed coupling delayed via hybrid nonlinear and impulsive control. Chaos Solitons Fractals 2018, 114, 381-393. [CrossRef]

14. Rao, R.; Zhong, S.; Wang, X. Stochastic stability criteria with LMI conditions for Markovian jumping impulsive BAM neural networks with mode-dependent time-varying delays and nonlinear reaction-diffusion. Commun. Nonlinear Sci. Numer. Simul. 2014, 19, 258-273. [CrossRef]

15. Li, X.; Chen, W.; Zheng, W.; Wang, Q. Instability and Unboundedness Analysis for Impulsive Differential Systems with Applications to Lurie Control Systems. Int. J. Control Autom. Syst. 2018, 16, 1521-1531. [CrossRef]

16. Yang, H.; Wang, X.; Zhong, S. Synchronization of nonlinear complex dynamical systems via delayed impulsive distributed control. Appl. Math. Comput. 2018, 320, 75-85. [CrossRef]

17. Wang, X.; She, K.; Zhong, S. Pinning cluster synchronization of delayed complex dynamical networks with nonidentical nodes and impulsive effects. Nonlinear Dyn. 2017, 8, 2771-2782. [CrossRef]

18. Li, X.; Shen, J.; Rakkiyappan, R. Persistent impulsive effects on stability of functional differential equations with finite or infinite delay. Appl. Math. Comput. 2018, 329, 14-22. [CrossRef]

19. Li, X.; Li, P.; Wang, Q. Input/output-to-state stability of impulsive switched systems. Syst. Control Lett. 2018, 116, 1-7. [CrossRef]

20. Li, X.; Shen, J.; Akca, H. Comparison principle for impulsive functional differential equations with infinite delays and applications. Commun. Nonlinear Sci. Numer. Simul. 2018, 57, 309-321. [CrossRef]

21. Song, Q.; Cao, J. Dynamics of bidirectional associative memory networks with distributed delays and reaction-diffusion terms. Nonlinear Anal. Real World Appl. 2007, 8, 345-361. [CrossRef]

22. Zhu, Q.; Li, X.; Yang, X. Exponential stability for stochastic reaction-diffusion BAM neural networks with time-varying and distributed delays. Appl. Math. Comput. 2011, 217, 6078-6091. [CrossRef]

23. Zeng, D.; Pu, Z.; Zhang, R.; Zhong, S.; Liu, Y.; Wu, G. Stochastic reliable synchronization for coupled Markovian reaction-diffusion neural networks with actuator failures and generalized switching policies. Appl. Math. Comput. 2019, 357, 88-106. [CrossRef]

24. Song, Q.; Cao, J.; Zhao, Z. Periodic solutions and its exponential stability of reaction-diffusion recurrent neural networks with continuously distributed delays. Nonlinear Anal. Real World Appl. 2006, 7, 65-80. [CrossRef]

25. Rao, R.; Zhong, S.; Pu, Z. Fixed point and p-stability of T-S fuzzy impulsive reaction-diffusion dynamic neural networks with distributed delay via Laplacian semigroup. Neurocomputing 2019, 335, 170-184. [CrossRef]

26. Hodara, P.; Papageorgiou, I. Poincare-Type Inequalities for Compact Degenerate Pure Jump Markov Processes. Mathematics 2019, 7, 518 [CrossRef]

27. Zhang, R.; Zeng, D.; Park, J.; Zhong, S. A New Approach to Stochastic Stability of Markovian Neural Networks With Generalized Transition Rates. IEEE Trans. Neural Netw. Learn. Syst. 2019, 30, 499-510. [CrossRef] [PubMed]

28. Li, X.; Rakkiyappan, R.; Sakthivel, N. Non-Fragile Synchronization Control For Markovian Jumping Complex Dynamical Networks With Probabilistic Time-Varying Coupling Delays. Asian J. Control 2015, 17, 1678-1695. [CrossRef]

29. Zhang, R.; Liu, X.; Zeng, D.; Zhong, S.M.; Shi, K.B. A novel approach to stability and stabilization of fuzzy sampled-data Markovian chaotic systems. Fuzzy Sets Syst. 2018, 344, 108-128. [CrossRef] 
30. Ding, Y.; Zhong, S.; Long, S. Asymptotic stability in probability of singular stochastic systems with Markovian switchings. Int. J. Robust Nonlinear Control 2017, 27, 4312-4322. [CrossRef]

31. Li, X.; Rakkiyappan, R. Delay-dependent global asymptotic stability criteria for stochastic genetic regulatory networks with Markovian jumping parameters. Appl. Math. Model. 2012, 36, 1718-1730. [CrossRef]

32. Rao, R. Delay-Dependent exponential stability for nonlinear reaction-diffusion uncertain Cohen-Grossberg neural networks with partially known transition rates via Hardy-Poincare inequality. Chin. Ann. Math. Ser. B 2014, 35, 575-598. [CrossRef]

33. Rao, R.; Zhong, S.; Pu, Z. On the role of diffusion factors in stability analysis for p-Laplace dynamical equations involved to BAM Cohen-Grossberg neural network. Neurocomputing 2017, 223, 54-62. [CrossRef]

34. Pu, Z.; Rao, R. Delay-dependent LMI-based robust stability criterion for discrete and distributed time-delays Markovian jumping reaction-diffusion CGNNs under Neumann boundary value. Neurocomputing 2016, 171, 1367-1374. [CrossRef]

35. Temam, R. Infinite Dimensional Dynamical Systems in Mechanics and Physics; Springer: NewYork, NY, USA; Berlin, Germany, 1998.

36. Rao, R.; Hang, J.; Zhong, S. Global Exponential Stability of Reaction-Diffusion BAM Neural Networks. J. Jilin Univ. (Sci. Ed.) 2012, 50, 1086-1090. (In Chinese)

(c) 2019 by the author. Licensee MDPI, Basel, Switzerland. This article is an open access article distributed under the terms and conditions of the Creative Commons Attribution (CC BY) license (http:/ / creativecommons.org/licenses/by/4.0/). 